This item was submitted to Loughborough's Research Repository by the author.

Items in Figshare are protected by copyright, with all rights reserved, unless otherwise indicated.

\title{
Impact of inertia emulation control of grid-scale BESS on power system frequency response
}

PLEASE CITE THE PUBLISHED VERSION

http://dx.doi.org/10.1109/ICSAE.2016.7810198

PUBLISHER

(C) IEEE

VERSION

AM (Accepted Manuscript)

LICENCE

CC BY-NC-ND 4.0

\section{REPOSITORY RECORD}

Alhejaj, Samir M., and Francisco M. Gonzalez-Longatt. 2019. "Impact of Inertia Emulation Control of Gridscale BESS on Power System Frequency Response”. figshare. https://hdl.handle.net/2134/23097. 


\section{Impact of Inertia Emulation Control of Grid-Scale BESS on Power System Frequency Response}

\author{
Samir M. Alhejaj \\ Wolfson School of Mechanical, \\ Electrical \& Manufacturing Engineering \\ Loughborough University \\ Loughborough, UK \\ s.m.alhejaj@lboro.ac.uk
}

\author{
Francisco M. Gonzalez-Longatt \\ Wolfson School of Mechanical, \\ Electrical \& Manufacturing Engineering \\ Loughborough University \\ Loughborough, UK \\ fglongatt@fglongatt.org
}

\begin{abstract}
Battery Energy Storage System (BESS) becomes important for many applications, including frequency regulation and control. This paper presents a comprehensive analysis of all the electro-mechanical-chemical variables involved in improving the system frequency response. It also evaluates the impact of including inertial emulation control of grid-scale BESS on the system frequency response of classical transmission systems. In order to investigate this impact, several simulations case studies are carried out, with different sudden generation outages and load disturbances with different power supply capacities. All case studies are combined with time-domain events to inspect the dynamic behavior of the frequency response of grid-scale battery. The purpose of this paper is to investigate the inertial response to design a set of algorithms to control the output of the inertial controller.
\end{abstract}

Keywords-Frequency Response, Inertia Emulation Control, Grid-Scale BESS.

\section{INTRODUCTION}

In recent years, the power system has undergone a massive transformation in terms of changing infrastructure and introducing control technologies. The foremost consideration of this change is principally represented by replacing some of the Conventional Power Plant (CPP) that is predominantly operated by fossil-fuel with environmental friendly energy resources, such as Wind Power Plant (WPP) and photovoltaic and solar power. This transformation has brought considerable ecological and economic benefits; however, this does not come without a price. Normally, CPP is more controllable and convenient for power system stability in contrast to new generation resources, such as Renewable Energy Resources (RES). Most RES is characterized by their intermittency and uncontrollable nature, and they mostly depend on the weather forecast to provide electric power. CPP can provide frequency response to preserve the balance between the demand and load, which is essential for power system stability.

Frequency response is classified, based on the time of reaction, into three broad categories: Inertial Response (IR), Primary Frequency Control (PFC), Secondary Frequency Control tertiary control. PFC and secondary frequency control are provided by two main control techniques: turbine governor and Automatic Generation Control (AGC), respectively. However, IR inherently exists in the system and is part of it. Because it principally arises due to the physical phenomena of kinetic energy generated and stored in all rotating parts of load motors and synchronous generators. If any disturbance occurs, power unbalance in the power system unpreventably follows; this kinetic energy is released in parts of a second to arrest this change and prevent the system frequency going under limits. With the help of previously mentioned system control strategies, the inertial response can bring the frequency to its scheduled value in few seconds to minutes. Therefore, the grid inertial frequency response is a fast frequency response, and it is an important factor in power system stability, especially during the early stage of system frequency disturbance.

With introductory of RES, these three control strategies become less effective. IR is mainly affected, since most RES are connected to the grid through electronic converters and provide significantly no IR to the grid [1]. New control techniques are introduced to use with such intermittent energy sources. Control strategies, such as Maximum Power Point Tracking (MPPT) and inertial emulation techniques, use ESS, mainly BESS. The influence of different ESS, specifically BESS and flywheels, on large power system frequency responses were studied by [2]. In another paper, [3] develops two control strategies, Coordinated Constant Droop Control (CCDC) and Coordinated Adoptive Droop Control (CADC), to emulate the grid inertial response with the aid of BESS and Flywheels.

The main benefits of using BESS for frequency control comes from three main points. First, it replaces the fast start reserves gas turbine, preserved to be used if any disturbance occurs. Second, its fast response would replace and substitute the online reserve generators; therefore, it works as a supplementary PFC. It supports the system inertial response and acts as an alternative to the declined inertia. Current breakthrough in developing BESS promises extensive use in coming years.

In this paper, sensibility analysis is used to examine how the parameters of BESS controller effect electro-mechanicalchemist variables related to the frequency response. This paper evaluates the impact of changing the gain of inertial emulation controller Hsyn of the grid-scale BESS on the system frequency. Many time-domain simulation case studies, using DIgSILENT PowerFactory, are applied to evaluate the system frequency response with changeable several values of Hsyn disturbances. The outcomes of this time-domain analysis study 
are to test and analyze different inertia emulation responses with different disturbance events. The results will be discussed to create plans to develop a control strategy to control the emulation inertia response for the BESS controller and test it in a real-time environment.

The paper is organized as follows: In Section II, an illustration about grid inertial response is given and how it is affected by the BESS parameters, mainly the inertia emulated the response of the battery in use. The section IV simulation process is discussed and a description of the results obtained during the simulation is presented. The paper is finalized by giving conclusions and references.

\section{BESS AND GRID INERTIAL RESPONSE}

Using any energy storage on a grid level application is possible by using a set of control techniques to synthesize the rotating mass and damping effect as in the synchronous generators. The strategy of controlling the Power Electronic Interface (PEI) of the grid converter (inverter) associated with any distributed energy source is called Virtual Synchronous generator (VSG). Therefore, the main three concepts that are adopted to simulate the behavior of the synchronous generator are to derive the rotor motion, governor response, and delay characteristic of the reactive power [4]. The concept of VSG can be applied on any ESS to synthesize the behavior of a synchronous generator and produce one or both active and reactive powers.

By ignoring the losses, the power balance for any grid is described by (1) as follows:

$$
P_{e}+P_{R E S}+P_{E S S}=P_{l}
$$

For simplicity of the analysis in this paper and since no RES is used, this equation can be simplified as in (2):

$$
P_{e}+P_{E S S}=P_{l}
$$

BESS, like other ESS, mainly comprises four main parts: an energy storage device (a battery), power-electronic converter or PEI, controller, and coupling power transformer. The battery is an ideal dc voltage source, whereas, PEI is twolevel Voltage Source converter (VSC) [4]. If the abc-dq transformation is perfectly synchronized with grid voltages, then the active power can be described as in (3):

$$
\Delta p_{s}=V_{d} \Delta i_{s}^{d}
$$

where $V_{d}$ and $i_{s}^{d}$ are the d-axis component of the grid voltages and VSC ac current, respectively. From the equation above, the active power of VSC can be controlled by $i_{s}^{d}$, which dynamic is described in (4):

$$
L \Delta \dot{\hat{\imath}}_{s}^{d}=-r \Delta i_{s}^{d}+2 \pi f L \Delta i_{s}^{q}+V_{d c} \Delta m^{d}-V_{d}
$$

where $f$ is the system frequency, $i_{s}^{q}$ is the q-axis component of the VSC as current, $m^{d}$ is the d-component of the modulation index, whereas $\mathrm{L}$ and $r$ are the inductance and resistance of the
VSC output filter. Therefore, a closed loop uses $i_{s}^{d}$ to control VSC as in (5)

$$
\begin{gathered}
\Delta m^{d}=\frac{1}{V_{d c}}\left\{\left(k_{s i}+r\right) \Delta i_{s r e f}-k_{s i} \Delta i_{s}^{d}-2 \pi f L \Delta i_{s}^{q}+\right. \\
\left.V_{d}\right\}
\end{gathered}
$$

where $k_{s i}$ is the current controller gain, $i_{s \text { ref }}$ is the d-axis current reference signal.

To control the above PEI, a virtual inertia controller is needed. Normally, to control the active power in PEI in inverse proportion to the derivative of the grid frequency, the control law for controlling the active power for PEI through optimal virtual inertia controller is described in (6)

$$
\Delta p_{\text {s ref }}=-k_{v i} k_{r}^{2} f_{\circ} \dot{\Delta f}
$$

where $k_{v i}$ is the virtual inertia added to the system, $k_{r}=2 \pi$ / $n_{p}$ is the conversion factor between the rotor speed and grid frequency, with $n_{p}$ being the number of poles of the machine, $f \circ$ is the nominal grid frequency. Fig. 1 shows the PEI and the optimal virtual inertia controller.

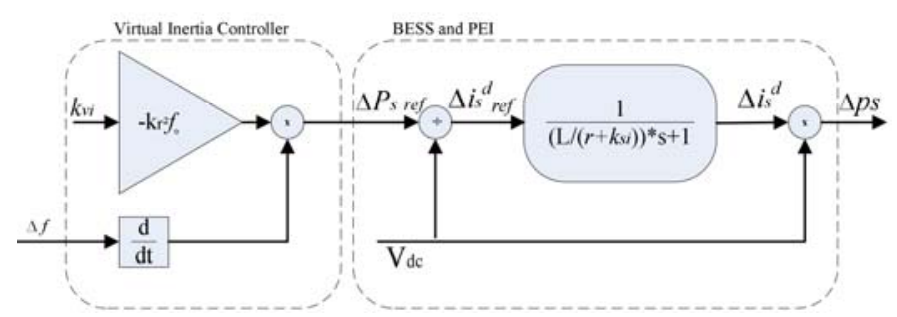

Fig. 1. PEI and Virtual Inertial Response Control Loops

\section{TEST SYSTEM}

\section{A. Grid and BESS Models}

A grid is modeled using DIgSILENT PowerFactory to demonstrate the simulation and obtain the results of the inertial response and BESS parameters. Fig. 2 shows an illustrative test model for a power grid comprising two synchronous generators, G1 and G2. Both generators are connected to bus number 1 . They have an identical power capacity of 500 MVA and voltage $230 \mathrm{kV}$ rating; however, G1 is set as a reference machine. The grid is set to operate with frequency $50 \mathrm{~Hz}$.

To simulate a load increment disturbance event, an inductive load of $100 \mathrm{MW}$ and power factor 0.99 is connected to bus number 2. A transmission line of $0.5 \mathrm{~km}$ length is connected to the main buses 1 and 2. BESS is connected through the electronic converter and a coupling transformer into bus number 2 to provide the necessary inertial support to the grid.

Fig. 2 shows the grid main operational parameters at steady-state conditions after running the load flow analysis. From the figure, G1 supplies exactly three-quarters of the active power requested by the load, whereas the slack generator G1 supplies about $25.9 \mathrm{MW}$ active power shared by the load (25 MW), BESS (0.1 MW), and finally, (0.9 MW) as losses. 


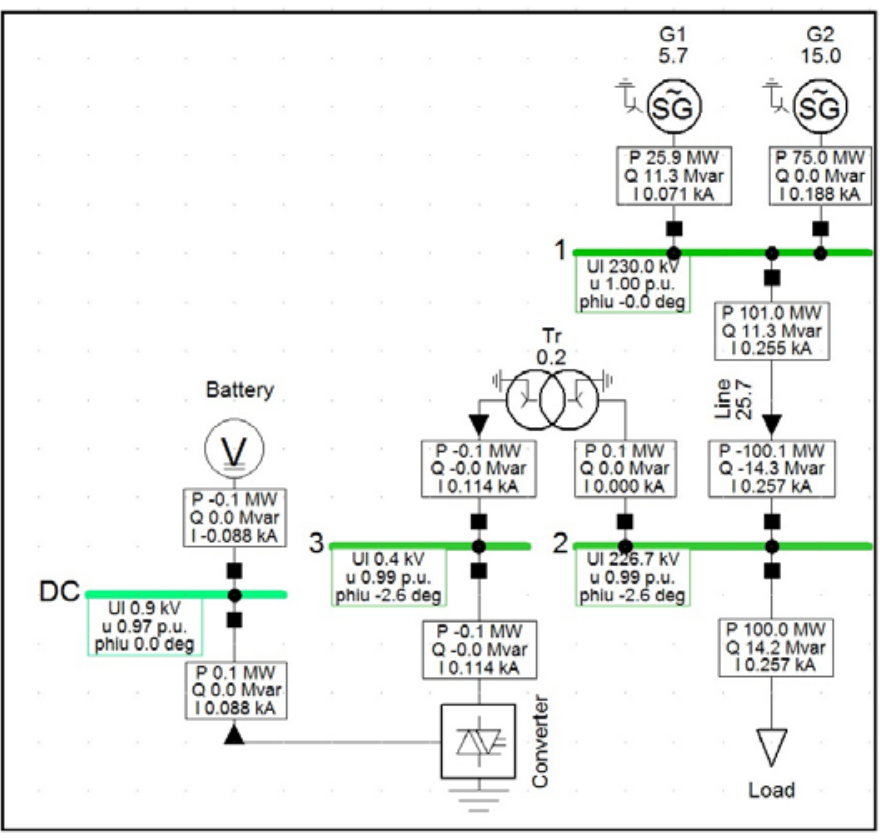

Fig. 2. Grid System of to generators, load, and BESS.

BESS System comprises of a generic battery model is described by [5] used as a dynamic battery for this study as in Fig. 3.
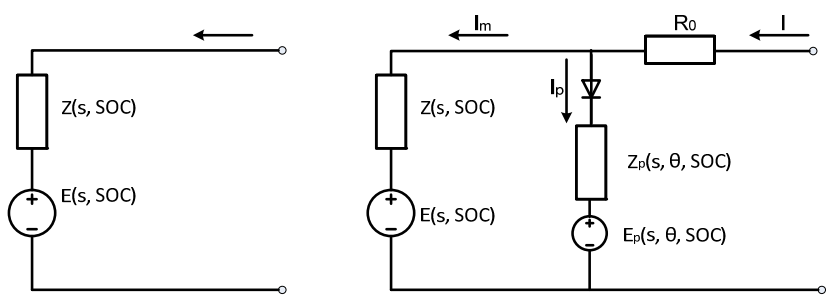

Fig. 3. Circuit equivalent model representative of an electric battery [5]

(a) Simple model without parasitic reaction and

(b) including the parasitic reaction effect.

The battery is connected to the DC bus with the following parameters as they are listed in Table I.

TABLE I.

BATTERY PARAMETERS

\begin{tabular}{|l|l|c|}
\hline \multicolumn{1}{|c|}{ Parameter } & \multicolumn{1}{c|}{ Value } & Unit \\
\hline SoC & 0.8 & - \\
\hline Cell Capacity & 1.2 & $\mathrm{Ah}$ \\
\hline Minimum Empty Cell Voltage & 12 & $\mathrm{~V}$ \\
\hline Maximum Full Cell Voltage & 13.85 & $\mathrm{~V}$ \\
\hline Number of Parallel Cell & 60 & - \\
\hline Number of Row Cell & 65 & - \\
\hline Nominal Voltage & 0.9 & $\mathrm{kV}$ \\
\hline Internal Resistance & 0.001 & $\mathrm{ohm}$ \\
\hline
\end{tabular}

A simple controller is used to emulate the inertial response in BESS as it appears in Fig. 4. The main parameter $H_{s y n}$ is set dynamically from 0.01 to 500 in step 10 in each simulation case study as described in the next section.

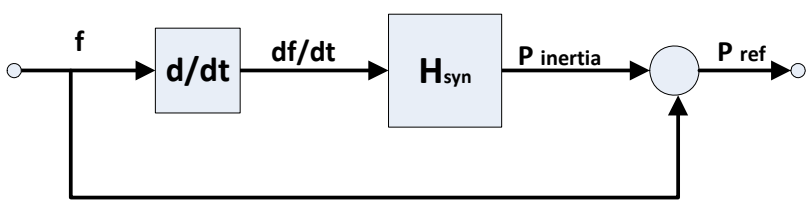

Fig. 4. Inertial Response controller.

Also, charge and PV controllers are also used to control the battery operation. The charge controller is used to control the charge and SOC for the battery, whereas PV controller is used for controlling the maximum and minimum $I_{d}$ and $I_{q}$ current, so BESS is controlled as VSG as described in the previous section.

\section{B. Simulation Case Studies and results}

Two case studies include generator outage (G2) and sudden load disturbances are carried out in this process. Both studies are discussed in detail in the following two subsections. In each case study, three scenarios are carried out with different generation capacity. Therefore, G2 is set to operate under 25, 50 , and $75 \mathrm{MW}$ respectively for each of the three scenarios of each case study. Also, the gain of the inertial emulation controller Hsyn of grid-scale BESS is changed from 0.001 to 500 in step 10 for each test. To simulate the dynamic frequency response and BESS behavior, a time-domain simulation last for 7 sec. and disturbance event is carried out at the first second after the start of the simulation for each test.

\section{1) Case Study 1 - Generator Outage}

In the first case study, a generator outage is set as disturbance event at the first second at the start of the simulation. Three scenarios with different generation capacities are considered. With each dispatched active power, 3 sets of different load demand level are tested. Taking the first scenario as an example, the demand for active power is set to 25,50 , and $75 \mathrm{MW}$ for each test. Table II lists all the grid parameters used in this simulation.

TABLE II. CASE StUdy 1 - OUtAGE

\begin{tabular}{|c|c|c|}
\hline \multirow{2}{*}{$\begin{array}{l}\text { Scenario } \\
\text { Number }\end{array}$} & Scenario & Test \\
\hline & $\begin{array}{c}\text { G2 dispatched active } \\
\text { power (MW) }\end{array}$ & $\begin{array}{l}\text { Load Demand } \\
(M W)\end{array}$ \\
\hline \multirow{3}{*}{1} & \multirow{3}{*}{75} & 75 \\
\hline & & 50 \\
\hline & & 25 \\
\hline \multirow{3}{*}{2} & \multirow{3}{*}{50} & 75 \\
\hline & & 50 \\
\hline & & 25 \\
\hline \multirow{3}{*}{3} & \multirow{3}{*}{25} & 75 \\
\hline & & 50 \\
\hline & & 25 \\
\hline
\end{tabular}


Fig. 5, 6, and 7 show the graphs for the main electric (Power, DC voltage), electro-mechanical (system frequency) and chemical (SOC) parameters for the system for three tests.

Fig. 5 shows the system parameters with the loss of $75 \mathrm{MW}$ power, whereas the load is $75 \mathrm{MW}$. Most of this lost power is compensated by G1 and BESS. The figure indicates that there is almost no inertia response contribution from the battery immediately after the sudden disturbance when Hsyn is equal to $(0.01)$, but the inertia contribution (active power) increases when the value of Hsyn is set higher. The steady-state frequency $\left(f_{s s}\right)$ is settled at a value less than $(49.6 \mathrm{~Hz})$ for all Hsyn values.
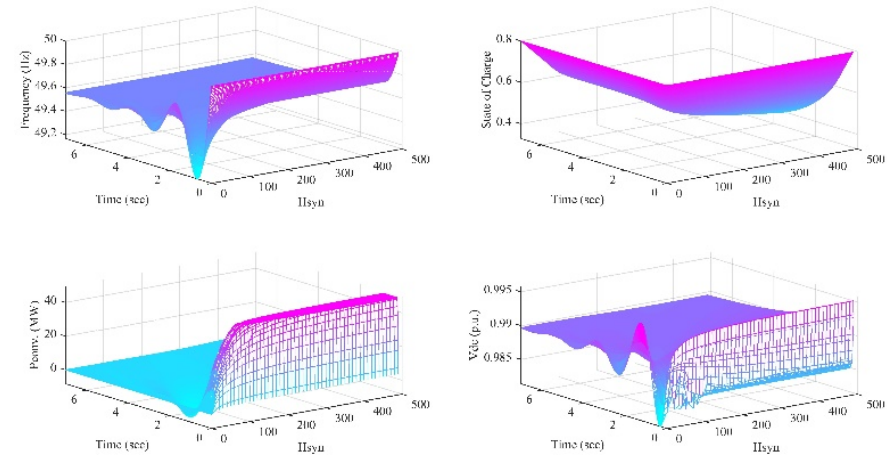

Fig. 5. Generator $75 \mathrm{MW}$ outage and load demand $75 \mathrm{MW}$.

Fig. 6 shows the system parameters after the loss of $75 \mathrm{MW}$ but with a load is set to $25 \mathrm{MW}$ only. It is clear that the contribution of the BESS inertial response to the power grid is less than the previous test. Also, the steady-state values for the frequency are reached to higher values when Hsyn is increased.
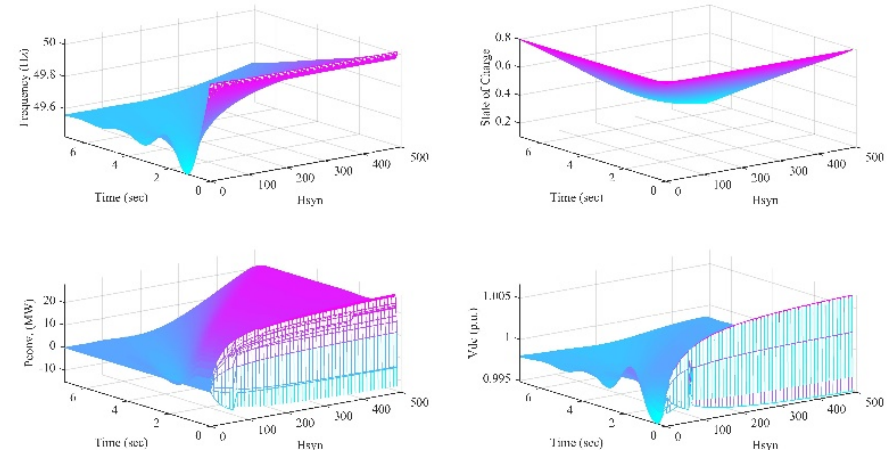

Fig. 6. Generator $25 \mathrm{MW}$ outage and load demand $75 \mathrm{MW}$.

Similar analysis is carried by [2], showing the same results, however, no synthetic inertial response is implemented to show the dynamic behavior of the frequency stability of the system. This paper shows the system frequency response for different inertial response and how it reacts with BESS.
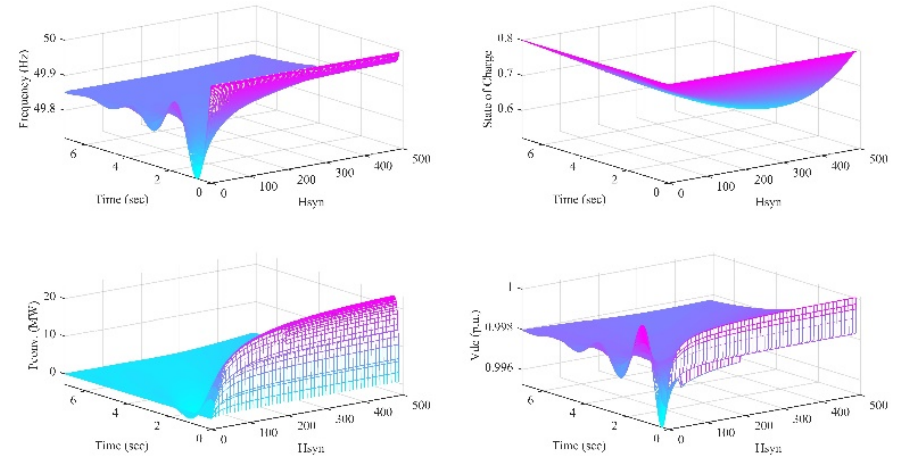

Fig. 7. Generator $25 \mathrm{MW}$ outage and load demand $25 \mathrm{MW}$.

\section{2) Case Study 2 - Load Increment}

In the second case study, three sudden load events with an increment of $25 \%, 50 \%$, and $75 \%$ are created and tested with different dispatched active power values supplied by G2 (no outage in this case) as listed in Table III.

TABLE III. CASE STUdy 2 - SudDEN LOAD INCREMENT

\begin{tabular}{|c|c|c|}
\hline \multirow{2}{*}{$\begin{array}{l}\text { Scenario } \\
\text { Number }\end{array}$} & Scenario & Test \\
\hline & $\begin{array}{c}\text { G2 dispatched active } \\
\text { power }(M W)\end{array}$ & Load Demand \\
\hline \multirow{3}{*}{1} & \multirow{3}{*}{75} & $75 \%$ \\
\hline & & $50 \%$ \\
\hline & & $25 \%$ \\
\hline \multirow{3}{*}{2} & \multirow{3}{*}{50} & $75 \%$ \\
\hline & & $50 \%$ \\
\hline & & $25 \%$ \\
\hline \multirow{3}{*}{3} & \multirow{3}{*}{25} & $75 \%$ \\
\hline & & $50 \%$ \\
\hline & & $25 \%$ \\
\hline
\end{tabular}

Fig. 8, 9, and 10 show three selected graphs for this case study. From the graphs, it can be noticed that load increment does not cause frequency excursions as in the first case study. The reason for that is obvious, since the two generators, G2 and G2, still have a large contribution to the active power with support from BESS.

The obtained results show how the frequency improves when the inertia emulation controller Hsyn of the grid-scale BESS is increased; however, discharging Pconv is affected during this period of time. It is also noticed that the SOC has a deeper voltage drop on the battery cells $(V d c)$. 

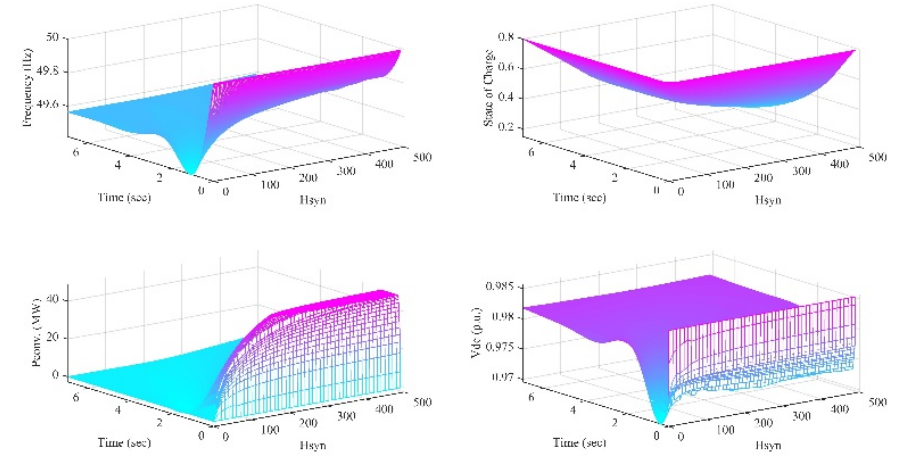

Fig. 8. Generator $75 \mathrm{MW}$ dispatch and load $75 \%$ increment.
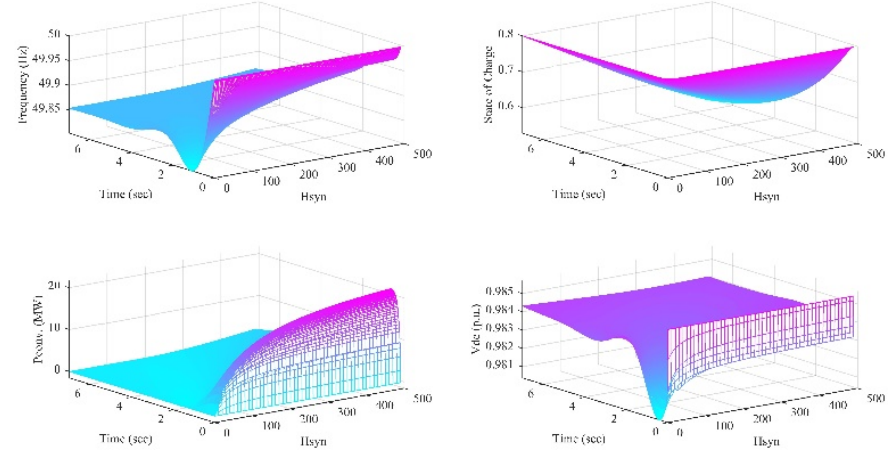

Fig. 9. Generator $75 \mathrm{MW}$ dispatch and load $25 \%$ increment.
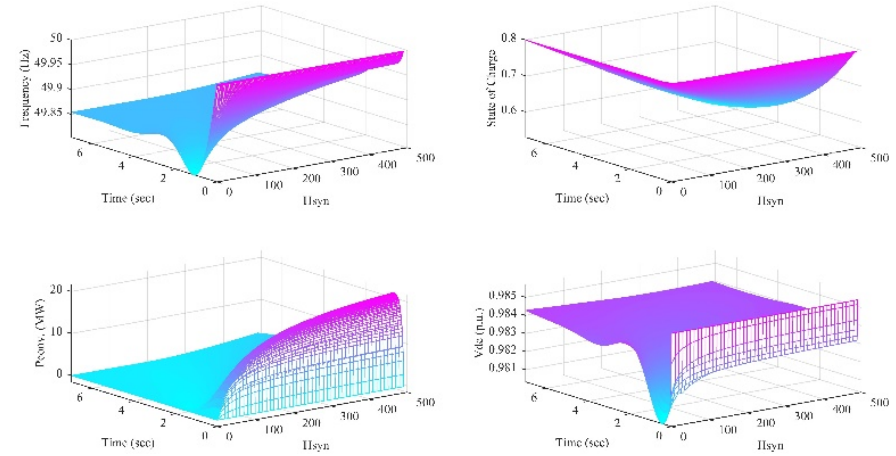

Fig. 10. Generator $25 \mathrm{MW}$ dispatch and load 25\% increment.
The graphs for all tests demonstrate the frequency does not return to its nominal value, because no other ancillary control services, such as PFC and secondary frequency control, are installed in this simulation model.

\section{CONCLUSIONS}

This paper explores the impact of using BESS at the gridscale level and how this will affect the inertial response if unbalance event occurs. Case studies are developed to study the effectiveness of using BESS on the inertial frequency response, using DIgSILENT PowerFactory simulation software. A simple controller is used to control the gain of the inertial response of the grid-scale battery. Many disturbance events are created in a time-domain manner to simulate the different power unbalance in the grid. The data from each simulation are gathered and analyzed in Matlab. The results show the main system parameters are mostly affected by this unbalance and responsible for the rate of change of the frequency, synchronous generator speed (system frequency), SOC, converter power, and dc voltage of the battery. Graphs are produced for these parameters. The study has revealed the emulated inertial response should be controlled to support the system frequency and inertial response. Therefore, algorithms based on optimization technique create an optimal and adaptive response, based on real-time accurate measurement.

\section{REFERENCES}

[1] F. Gonzalez-Longatt, E. Chikuni, W. Stemmet, K. Folly, "Effects of the synthetic inertia from wind power on the total system inertia after a frequency disturbance," Power Engineering Society Conference and Exposition in Africa (PowerAfrica), IEEE, pp. 1-7, 2012.

[2] A. Adrees, J. Song, J. V. Milanović, "The influence of different storage technologies on large power system frequency response," IEEE 8th International Power Electronics and Motion Control Conference (IPEMC-ECCE Asia), pp. 257 - 263, 2016.

[3] S. Sami, M. Cheng, J. Wu, "Modelling and control of multi-type gridscale energy storage for power system frequency response," IEEE 8th International Power Electronics and Motion Control Conference (IPEMC-ECCE Asia), pp. 269 - 273, 2016.

[4] M. Rashid, Power Electronics Handbook, Pensacola: Elsevier , 2011.

[5] J. Meng, X. Shi, Y. Wang, Ch. Fu, "A Virtual synchronous generator control strategy for distributed generation," China International Conference on Electricity Distribution (CICED), pp. 495 - 498, 2014.

[6] M. Chen, G.A. Rincon-More, "Accurate electrical battery model capable of predicting runtime and I-V performance," IEEE Transaction on Energy Conversion, vol. 21, no. 2, pp. 504-511, 2006. 